COMMUNICATIONS IN

ANALYSIS AND GEOMETRY

Volume 13, Number 1, 113-128, 2005

\title{
Orbifold Cohomology of the Symmetric Product
}

\author{
BERNARDO URIBE
}

\begin{abstract}
Chen and Ruan's orbifold cohomology of the symmetric product of a complex manifold is calculated. An isomorphism of rings (up to a change of signs) $H_{\text {orb }}^{*}\left(X^{n} / S_{n} ; \mathbb{C}\right) \cong H^{*}\left(X^{[n]} ; \mathbb{C}\right)$ between the orbifold cohomology of the symmetric product of a smooth projective surface with trivial canonical class $X$ and the cohomology of its Hilbert scheme $X^{[n]}$ is obtained, yielding a positive answer to a conjecture of Ruan.
\end{abstract}

\section{Introduction.}

String theorists (see [3]) proposed an Euler characteristic for orbifolds that are global quotients by the action of a finite group; this number matched the Euler characteristic of equivariant $K$-theory (see [1]). For these global quotients, Zaslow [9] worked out an additive orbifold cohomology and later on, Chen and Ruan [2] and Ruan [7] generalized this construction to a general orbifold. Motivated by the study of quantum cohomology, they developed a new ring structure for the cohomology of orbifolds. This ring structure is different to the ones obtained by other equivariant cohomology theories such as equivariant K-theory or Bredon Cohomology, and for the case of the symmetric product it will be explicitly calculated in this paper. For $X$ an algebraic surface, it is known that the Hilbert scheme $X^{[n]}$ of points of length $n$ is a crepant resolution of $\operatorname{Symm}_{n}(X)$; Ruan [7] conjectured that if $X^{[n]}$ had hyperkähler structure then its cohomology and the orbifold cohomology of $\operatorname{Symm}_{n}(X)$ should be isomorphic as rings. From the calculation of the orbifold cohomology of $\mathbb{C}^{n} / G$, with $G$ a finite subgroup of $G L(n, \mathbb{C})$, Ruan obtained the orbifold cohomology of $\operatorname{Symm}_{n}\left(\mathbb{C}^{2}\right)$ which was matched with the cohomology of $\left(\mathbb{C}^{2}\right)^{[n]}$ obtained by Lehn and Sorger [6]. The same authors, in the case of a smooth projective surface with trivial canonical class $X$ calculated the ring structure of the cohomology of $X^{[n]}$ [5]. Using the explicit calculation of the orbifold cohomology of $\operatorname{Symm}_{n}(X)$, when $X$ is a smooth projective surface with trivial canonical class, an isomorphism (up to a change of sign) between the orbifold cohomology of $\operatorname{Symm}_{n}(X)$ and the cohomology of $X^{[n]}$ is obtained. 
The organization of the paper is as follows, in the first section the basic definitions of orbifold cohomology are summarized, and in the second section the cohomology ring structure of the symmetric product is explained. After obtaining the results in this paper, I was notified that they had also been independently obtained by Fantechi and Göttsche [4].

Last but not least, I would like to express my deepest gratitude to E. Lupercio, M. Poddar, A. Adem and Y. Ruan who shared with me insightful ideas through informal meetings and especially to the latter two who introduced me to the subject.

\section{Preliminaries.}

\subsection{Orbifold Cohomology.}

An $n$-dimensional complex orbifold is a topological space $Y$ together with an open cover $\mathcal{U}$ such that every open set $U_{i} \in \mathcal{U}$ is homeomorphic to $\mathbb{C}^{n} / \Gamma_{i}$ where $\Gamma_{i}$ is a finite subgroup of $\mathrm{GL}_{n}(\mathbb{C})$. The gluing conditions for this cover are subtle and can be found in the papers of Chen and Ruan [2] and of Ruan [7] (their notation will be followed). Here we will concentrate on orbifolds that could be realized as global quotients, i.e. $Y=X / G$ where $X$ is a complex manifold and $G$ is a finite group.

The twisted sectors are the sets

$$
Y_{(\mathbf{g})}=X^{\mathbf{g}} / C(\mathbf{g})
$$

where $X^{\mathbf{g}}=X^{g_{1}} \cap \cdots \cap X^{g_{k}}$ for $X^{g_{i}}$ the fixed point set of the action of $g_{i}$, $T_{k}$ is the set of conjugacy classes of $k$-tuples $\mathbf{g}=\left(g_{1}, \ldots, g_{k}\right)$ of elements in $G$ and $C(\mathbf{g})=C\left(g_{1}\right) \cap \cdots \cap C\left(g_{k}\right)$, with $C\left(g_{i}\right)$ the centralizer of $g_{i}$ in $G$. The multisectors $\widetilde{\Sigma_{k} Y}$ are the disjoint union of the twisted sectors, i.e.

$$
\widetilde{\Sigma_{k} Y}=\bigsqcup_{(\mathbf{g}) \in T_{k}} Y_{(\mathbf{g})}
$$

It is worth pointing out that the twisted sectors $Y_{(\mathbf{g})}$ may contain several connected components of different dimensions, this fact is taken into account in what follows.

Let's now consider the natural maps between multi-sectors; the evaluation maps $e_{i_{1}, \ldots, i_{l}}: \widetilde{\Sigma_{k} Y} \rightarrow \widetilde{\Sigma_{l} Y}$ defined by $e_{i_{1}, \ldots, i_{l}}\left(x,\left(g_{1}, \ldots, g_{k}\right)\right) \mapsto$ $\left(x,\left(g_{i_{1}}, \ldots, g_{i_{l}}\right)\right)$ and the involutions $I: \widetilde{\Sigma_{k} Y} \rightarrow \widetilde{\Sigma_{k} Y}$ defined by $I(x,(\mathbf{g})) \mapsto$ $\left(x,\left(\mathbf{g}^{-\mathbf{1}}\right)\right)$ where $\mathbf{g}^{\mathbf{1}}=\left(g_{1}^{-1}, \ldots, g_{k}^{-1}\right)$. 
To define the orbifold cohomology group we need to add a shifting to the cohomology of the twisted sectors, and for that we make use of the complex structure $J$; recall that $J$ is a smooth section of $\operatorname{End}(T Y)$ such that $J^{2}=-I d$.

For $p=[x] \in Y$ with $x \in X$ and $G_{x} \subset G$ the isotropy group at $x$, the complex structure gives rise to a faithful representation $\rho_{x}: G_{x} \rightarrow \mathrm{GL}_{n}(\mathbb{C})$ that for every $g \in G_{x}$ could be diagonalized as

$$
\operatorname{diag}\left(e^{2 \pi \frac{m_{1, g}}{m_{g}}}, \ldots, e^{2 \pi \frac{m_{n, g}}{m_{g}}}\right)
$$

where $m_{g}$ is the order of $g$ in $G_{i}$ and $0 \leq m_{j, g}<m_{g}$. We define a function $\iota: \widetilde{\Sigma_{1} Y} \rightarrow \mathbb{Q}$ by

$$
\iota(p,(g))=\sum_{j=1}^{n} \frac{m_{j, g}}{m_{g}}
$$

that is well defined because it only depends on the orbit of $x$ in $X$ and the conjugacy class of $g$. It is easy to see that it is locally constant, hence we call it $\iota_{(g)}$, the degree shifting number in each sector $Y_{(g)}$ (here we make an abuse of notation because this number may be different in each connected component, this will not cause any truoble). It is an integer if and only if $\rho_{x}(g) \in \mathrm{SL}_{n}(\mathbb{C}) ;$ and

$$
\iota_{(g)}+\iota_{\left(g^{-1}\right)}=\operatorname{rank}\left(\rho_{x}(g)-I\right)
$$

is the complex codimension of $Y_{(g)}$ i.e. $n-\operatorname{dim}_{\mathbb{C}} Y_{(g)}$.

Definition 2.1.1. The orbifold cohomology groups are defined as

$$
H_{\text {orb }}^{d}(Y ; \mathbb{C})=\bigoplus_{(g) \in T_{1}} H^{d-2 \iota_{(g)}}\left(Y_{(g)} ; \mathbb{C}\right)
$$

\subsection{Poincaré Duality.}

Let's assume the orbifold $Y$ is closed and recall the map $I: Y_{(g)} \rightarrow Y_{\left(g^{-1}\right)}$ defined by $(p,(g)) \mapsto\left(p,\left(g^{-1}\right)\right)$ with $I^{2}=I d$.

Poincaré Duality for the orbifold cohomology is as follows:

For any $0 \leq d \leq 2 n$, the pairing

$$
<>_{\text {orb }}: H_{\text {orb }}^{d}(Y ; \mathbb{C}) \otimes H_{\text {orb }}^{2 n-d}(Y ; \mathbb{C}) \rightarrow \mathbb{C}
$$


defined by the direct sum of

$$
<>_{\text {orb }}^{(g)}: H^{d-2 \iota(g)}\left(Y_{(g)} ; \mathbb{C}\right) \otimes H^{2 n-d-2 \iota\left(g^{-1}\right)}\left(Y_{\left(g^{-1}\right)} ; \mathbb{C}\right) \rightarrow \mathbb{C}
$$

where

$$
<\alpha, \beta>_{\text {orb }}^{(g)}:=\int_{Y_{(g)}} \alpha \wedge I^{*}(\beta)
$$

is nondegenerate and $\alpha \in H^{d-2 \iota(g)}\left(Y_{(g)} ; \mathbb{C}\right), \beta \in H^{2 n-d-2 \iota}\left(g^{-1}\right)\left(Y_{\left(g^{-1}\right)} ; \mathbb{C}\right)$.

When restricted to the non-twisted sector this is the ordinary Poincaré pairing.

\subsection{Orbifold Cup Product.}

The orbifold cup product relies on the construction of a obstruction bundle over the twisted sector $Y_{(\mathbf{g})}$ where $(\mathbf{g})=\left(g_{1}, g_{2}, g_{3}\right) \in T_{3}$ is the conjugacy class of the triple $\left(g_{1}, g_{2}, g_{3}\right)$ with $g_{1} g_{2} g_{3}=1$, and $T_{3}^{0}$ is the set of those conjugacy classes.

Let $e: Y_{(\mathrm{g})} \rightarrow Y$ be the evaluation map and $e^{*} T Y$ the pullback tangent bundle over $Y_{(\mathbf{g})}$. For $y \in Y_{(\mathrm{g})}$ its local group in $Y$ is $\Gamma^{\prime}$; clearly it contains the elements $g_{1}, g_{2}, g_{3}$ with the relations $g_{1} g_{2} g_{3}=1$ and $g_{i}^{k_{i}}$, where $k_{i}$ is the order of $g_{i}$. Let $\Gamma$ be the subgroup of $\Gamma^{\prime}$ generated by these three elements $g_{1}, g_{2}, g_{3}$; then $\Gamma$ acts on $e^{*} T Y$ while fixing $Y_{(\mathbf{g})}$.

For the orbifold Riemann sphere $S_{\left(x_{1}, x_{2}, x_{3}\right)}^{2}$ with only three orbifold points $x_{1}, x_{2}, x_{3} \in S^{2}$ each one being a cone point with angle $2 \pi / k_{i}$ (i.e. a neighborhood of $x_{i}$ is homeomorphic to $\mathbb{C} / \mathbb{Z}_{k_{i}}$ ) it is a result of Scott [8] that there exist a closed Riemann surface $\Sigma$ such that $\Gamma$ acts on it holomorphically and $(\Sigma, \Gamma, \pi)$ is a uniformizing system for $S_{\left(x_{1}, x_{2}, x_{3}\right)}^{2}$ (i.e. $\Sigma / \Gamma$ and $S_{\left(x_{1}, x_{2}, x_{3}\right)}^{2}$ are isomorphic as orbifolds). Now the group $\Gamma$ acts on both $H^{1}(\Sigma)$ and $e^{*} T Y$ when considering $H^{1}(\Sigma)$ as a trivial bundle over $Y_{(\mathbf{g})}$.

The obstruction bundle $E_{(\mathrm{g})}$ we require is the invariant part of $H^{1}(\Sigma) \otimes$ $e^{*} T Y$ under the action of $\Gamma$, i.e. $E_{(\mathbf{g})}=\left(H^{1}(\Sigma) \otimes e^{*} T Y\right)^{\Gamma}$. Let $c\left(E_{(\mathbf{g})}\right)$ be the Euler class of $E_{(\mathrm{g})}$ (which up to an exact form is independent of the connection) and recall the evaluation maps $e_{i}: Y_{(\mathrm{g})} \rightarrow Y_{\left(g_{i}\right)}$.

Definition 2.3.1. For $\alpha, \beta, \gamma \in H_{\text {orb }}^{*}(Y ; \mathbb{C})$ a three-point function is defined

$$
<\alpha, \beta, \gamma>_{\text {orb }}=\sum_{(\mathbf{g}) \in T_{3}^{0}} \int_{Y_{(\mathbf{g})}} e_{1}^{*} \alpha \cdot e_{2}^{*} \beta \cdot e_{3}^{*} \gamma \cdot c\left(E_{(\mathbf{g})}\right)
$$


and let the orbifold cup product be defined by the relation

$$
<\alpha \cup_{\text {orb }} \beta, \gamma>_{\text {orb }}=<\alpha, \beta, \gamma>_{\text {orb }}
$$

Lemma 2.3.2. For $\alpha \in H^{*}\left(Y_{\left(g_{1}\right)} ; \mathbb{C}\right)$ and $\beta \in H^{*}\left(Y_{\left(g_{2}\right)} ; \mathbb{C}\right)$ the cup product $\alpha \cup_{\text {orb }} \beta$ can be decomposed as a sum of its components in $H_{\text {orb }}^{*}(Y ; \mathbb{C})=$ $\bigoplus_{(g)} H^{*}\left(Y_{(g)} ; \mathbb{C}\right)$. So we have

$$
\alpha \cup_{\text {orb }} \beta=\sum_{\substack{\left(h_{1}, h_{2},\left(h_{1} h_{2}\right)^{-1}\right) \in T_{3}^{0} \\ h_{i} \in\left(g_{i}\right)}} p_{*}\left(e_{1}^{*} \alpha \cdot e_{2}^{*} \beta \cdot c\left(E_{(\mathbf{h})}\right)\right)
$$

where $p_{*}$ is the push-out of the evaluation map $p: Y_{\left(h_{1}, h_{2}\right)} \rightarrow Y_{\left(h_{1} h_{2}\right)}$.

Remark 2.3.3. In the definition of the three point function for the orbifold cup product an abuse of notation is being made. For $\alpha \in H^{*}\left(Y_{\left(g_{1}\right)} ; \mathbb{C}\right)$ we need to take into account the different elements conjugated to $g_{1}$, for $h_{1} \in\left(g_{1}\right), \phi: H^{*}\left(Y_{\left(g_{1}\right)} ; \mathbb{C}\right) \stackrel{\cong}{\rightrightarrows} H^{*}\left(Y_{\left(h_{1}\right)} ; \mathbb{C}\right)$ and let's denote by $\alpha_{h_{1}}:=\phi(\alpha)$. Then for $\beta \in H^{*}\left(Y_{\left(g_{2}\right)} ; \mathbb{C}\right)$ and $\gamma \in H^{*}\left(Y_{\left(g_{3}\right)} ; \mathbb{C}\right)$

$$
<\alpha, \beta, \gamma>_{\text {orb }}=\sum_{\substack{(\mathbf{h}) \in T_{3}^{0} \\ h_{i} \in\left(g_{i}\right)}} \int_{Y_{\left(h_{1}, h_{2}\right)}} e_{1}^{*} \alpha_{h_{1}} \cdot e_{2}^{*} \beta_{h_{2}} \cdot e_{3}^{*} \gamma_{h_{3}} \cdot c\left(E_{(\mathbf{h})}\right)
$$

The previous description becomes very complicated when calculations are tried out. In what follows, an equivalent description for the orbifold cohomology that simplifies calculations will be explained. The idea comes from the paper of Lehn and Sorger [5] and similar notation will be used.

Let $Y=X / G$ be an orbifold with $X$ a compact complex manifold. As before $X^{g}$ will denote the fixed point set of the action of $g$ on $X$.

The cohomology classes will be labeled by elements in $G$ and let the total $\operatorname{ring} A(X, G)$ be

$$
A(X, G):=\bigoplus_{g \in G} H^{*}\left(X^{g} ; \mathbb{C}\right) \times\{g\}
$$

Its group structure is the natural one and the ring structure that will be defined later will give us the orbifold cup product. The grading is the one in the orbifold cohomology, i.e.

$$
A^{d}(X, G)=\bigoplus_{g \in G} H^{d-2 \iota(g)}\left(X^{g} ; \mathbb{C}\right) \times\{g\}
$$


For $h \in G$ there is a natural map $h: X^{g} \rightarrow X^{h g h^{-1}}$ which can be extended to an action in $A(X, G)$ inducing an isomorphism

$$
\begin{aligned}
h: H^{*}\left(X^{g} ; \mathbb{C}\right) \times\{g\} & \rightarrow H^{*}\left(X^{h g h^{-1}} ; \mathbb{C}\right) \times\left\{h g h^{-1}\right\} \\
(\alpha, g) & \mapsto\left(\left(h^{-1}\right)^{*} \alpha, h g h^{-1}\right)
\end{aligned}
$$

The invariant part under the action of $G$ is isomorphic as a group to the orbifold cohomology,

\section{Lemma 2.3.4.}

$$
A(X, G)^{G} \cong \bigoplus_{(g)} H^{*}\left(X^{g} ; \mathbb{C}\right)^{C(g)} \cong H_{\text {orb }}^{*}(X / G ; \mathbb{C})
$$

Now some notation needs to be introduced in order to define the ring structure. This notation comes from [5] where a more detailed study of Frobenius algebras is done.

Definition 2.3.5. For $X^{\left\langle h_{1}, h_{2}\right\rangle}$, the fixed point set of $\left\langle h_{1}, h_{2}\right\rangle$, let

$$
\begin{gathered}
f^{h_{i},\left\langle h_{1}, h_{2}\right\rangle}: H^{*}\left(X^{h_{i}} ; \mathbb{C}\right) \rightarrow H^{*}\left(X^{\left\langle h_{1}, h_{2}\right\rangle} ; \mathbb{C}\right) \\
f_{\left\langle h_{1}, h_{2}\right\rangle, h_{i}}: H^{*}\left(X^{\left\langle h_{1}, h_{2}\right\rangle} ; \mathbb{C}\right) \rightarrow H^{*}\left(X^{h_{i}} ; \mathbb{C}\right)
\end{gathered}
$$

be the pull-back and the push-forward respectively of the diagonal inclusion map $X^{\left\langle h_{1}, h_{2}\right\rangle} \hookrightarrow X^{h_{i}}$ where $i=1,2,3$ and $h_{3}=\left(h_{1} h_{2}\right)$.

We need to make use of the obstruction bundle over $X^{\left\langle h_{1}, h_{2}\right\rangle}$; as $Y_{\left(h_{1}, h_{2}\right)}=X^{\left\langle h_{1}, h_{2}\right\rangle} / C\left(h_{1}, h_{2}\right)$ and taking the projection map $\pi: X^{\left\langle h_{1}, h_{2}\right\rangle} \rightarrow$ $X^{\left\langle h_{1}, h_{2}\right\rangle} / C\left(h_{1}, h_{2}\right)$ we will consider the Euler class of $\pi^{*}\left(E_{(\mathbf{h})}\right)$.

Definition 2.3.6. Let the product $A(X, G) \otimes A(X, G) \stackrel{\bullet}{\rightarrow} A(X, G)$ be defined by

$$
\left(\alpha, h_{1}\right) \cdot\left(\beta, h_{2}\right):=\left(f_{\left\langle h_{1}, h_{2}\right\rangle, h_{1} h_{2}}\left(f^{h_{1},\left\langle h_{1}, h_{2}\right\rangle}(\alpha) \cdot f^{h_{2},\left\langle h_{1}, h_{2}\right\rangle}(\beta) \cdot \pi^{*} c\left(E_{(\mathbf{h})}\right)\right), h_{1} h_{2}\right)
$$

whose three point function is

$$
\begin{aligned}
< & \left(\alpha, h_{1}\right),\left(\beta, h_{2}\right),\left(\gamma,\left(h_{1} h_{2}\right)^{-1}\right)> \\
& :=\int_{X^{\left\langle h_{1}, h_{2}\right\rangle}} f^{h_{1},\left\langle h_{1}, h_{2}\right\rangle}(\alpha) \cdot f^{h_{2},\left\langle h_{1}, h_{2}\right\rangle}(\beta) \cdot f^{\left(h_{1} h_{2}\right)^{-1},\left\langle h_{1}, h_{2}\right\rangle}(\gamma) \cdot \pi^{*} c\left(E_{(\mathbf{h})}\right)
\end{aligned}
$$


Lemma 2.3.7. The product $A(X, G) \otimes A(X, G) \stackrel{\circ}{\rightarrow} A(X, G)$ previously defined is $G$ equivariant.

This product induces a ring structure on the invariant group $A(X, G)^{G}$ which will match with the orbifold cup product. Thus $A(X, G)^{G}$ will inherit the properties of the orbifold cup product.

Proposition 2.3.8. The rings $H_{\text {orb }}^{*}(X / G ; \mathbb{C})$ and $A(X, G)^{G}$ are isomorphic.

Proof. From lemma 2.3.4 it is known that they are isomorphic as groups, but we need to have an explicit map

$$
\varphi: H_{\text {orb }}^{*}(X / G, \mathbb{C}) \rightarrow A(X, G)^{G} .
$$

For $\alpha \in H^{*}\left(Y_{\left(g_{1}\right)} ; \mathbb{C}\right)$ let $\alpha^{\prime} \in H^{*}\left(X^{g_{1}} ; \mathbb{C}\right)^{C\left(g_{1}\right)}$ be the pullback of $\alpha$ under the projection map $X^{g_{1}} \rightarrow Y_{\left(g_{1}\right)}=X^{g_{1}} / C\left(g_{1}\right)$. In the same way as in remark 2.3.3 let $\left(\alpha^{\prime}\right)_{h_{1}}:=\phi^{\prime}\left(\alpha^{\prime}\right)$ where $\phi^{\prime}: H^{*}\left(X^{g_{1}} ; \mathbb{C}\right) \cong H^{*}\left(X^{h_{1}} ; \mathbb{C}\right)$ and $h_{1} \in\left(g_{1}\right)$. As $\left(\alpha^{\prime}\right)_{h_{1}}=\left(\alpha_{h_{1}}\right)^{\prime}$ there will be no confusion in denoting it by $\alpha_{h_{1}}^{\prime}$.

The isomorphism of groups is clearly given by

$$
\varphi(\alpha):=\sum_{h_{1} \in\left(g_{1}\right)}\left(\alpha_{h_{1}}^{\prime}, h_{1}\right)
$$

and what is left to prove is that the triple functions give the same result.

For $\alpha \in H^{*}\left(Y_{\left(g_{1}\right)}, \mathbb{C}\right), \beta \in H^{*}\left(Y_{\left(g_{2}\right)}, \mathbb{C}\right)$ and $\gamma \in H^{*}\left(Y_{\left(g_{3}\right)}, \mathbb{C}\right)$ the triple function $<>_{G}$ induced by $<>$ in $A(X, G)^{G}$ is

$$
\begin{aligned}
& <\varphi(\alpha), \varphi(\beta), \varphi(\gamma)>_{G} \\
& =\frac{1}{|G|} \sum_{\substack{h_{i} \in\left(g_{i}\right) \\
h_{3}=\left(h_{1} h_{2}\right)^{-1}}} f_{X^{\left\langle h_{1}, h_{2}\right\rangle}}^{h_{1},\left\langle h_{1}, h_{2}\right\rangle}\left(\alpha_{h_{1}}^{\prime}\right) \cdot f^{h_{2},\left\langle h_{1}, h_{2}\right\rangle}\left(\beta_{h_{2}}^{\prime}\right) \cdot f^{h_{3},\left\langle h_{1}, h_{2}\right\rangle}\left(\gamma_{h_{3}}^{\prime}\right) \cdot \pi^{*} c\left(E_{(\mathbf{h})}\right)
\end{aligned}
$$

The group $G$ acts on the integral via conjugation, and it is clear that its value is invariant under this action. The action of $G$ by conjugation on the pairs $\left[h_{1}, h_{2}\right]$ has as stabilizer the group $C\left(h_{1}, h_{2}\right)$, thus we can exchange the set of pairs $\left[h_{1}, h_{2}\right]$ with $h_{i} \in\left(g_{i}\right)$ by the set of conjugacy classes of pairs $\left(h_{1}, h_{2}\right)$ multiplying by $|G| /\left|C\left(h_{1}, h_{2}\right)\right|$, the multiplicity of the integral ; hence

$$
\begin{aligned}
& <\varphi(\alpha), \varphi(\beta), \varphi(\gamma)>_{G} \\
& =\sum_{\substack{(\mathbf{h}) \in T_{3}^{0} \\
h_{i} \in\left(g_{i}\right)}} \frac{1}{\left|C\left(h_{1}, h_{2}\right)\right|} \int_{X^{\left\langle h_{1}, h_{2}\right\rangle}}^{f_{1},\left\langle h_{1}, h_{2}\right\rangle}\left(\alpha_{h_{1}}^{\prime}\right) \cdot f^{h_{2},\left\langle h_{1}, h_{2}\right\rangle}\left(\beta_{h_{2}}^{\prime}\right) \cdot f^{h_{3},\left\langle h_{1}, h_{2}\right\rangle}\left(\gamma_{h_{3}}^{\prime}\right) \cdot \pi^{*} c\left(E_{(\mathbf{h})}\right)
\end{aligned}
$$


Changing the set of integration and using that

$$
\int_{Y_{(g, h)}} \omega=\frac{1}{|C(g, h)|} \int_{X^{\langle g, h\rangle}} \pi^{*} \omega
$$

we get the desired equality

$$
\begin{aligned}
< & \varphi(\alpha), \varphi(\beta), \varphi(\gamma)>_{G} \\
& =\sum_{\substack{(\mathbf{h}) \in T_{3}^{0} \\
h_{i} \in\left(g_{i}\right)}} \int_{Y_{\left(h_{1}, h_{2}\right)}} e_{1}^{*}\left(\alpha_{h_{1}}\right) \cdot e_{2}^{*}\left(\beta_{h_{2}}\right) \cdot e_{3}^{*}\left(\gamma_{h_{3}}\right) \cdot c\left(E_{(\mathbf{h})}\right) \\
& =<\alpha, \beta, \gamma>_{\text {orb }}
\end{aligned}
$$

where one recalls that $\alpha_{h_{1}}^{\prime}=\pi^{*} \alpha_{h_{1}}$.

As the triple functions give the same result and the grading matches in both descriptions, the ring isomorphism follows. All the properties of the orbifold cup product proven in [2] apply to $A(X, G)^{G}$; this will be the description of orbifold cohomology that will be useful in the calculations that follow.

\section{Orbifold Cohomology of the Symmetric Product.}

In this section the previous description of the orbifold cup product of global quotients will be applied to the symmetric product. $X$ will be an even dimensional compact complex manifold $\operatorname{dim}_{\mathbb{C}} X=2 N$, and the orbifold in mind will be $X^{n} / S_{n}$ where the action of the symmetric group $S_{n}$ on $X^{n}$ is the natural one.

Some notation needs to be introduced.

Notation: For $\sigma, \rho \in S_{n}$, let $\Gamma \subset[n]:=\{1,2, \ldots, n\}$ be a set stable under the action of $\sigma$; we will denote by $\mathcal{O}(\sigma ; \Gamma)$ the set of orbits induced by the action of $\sigma$ in $\Gamma$. If $\Gamma$ is $\sigma$-stable and $\rho$-stable, $\mathcal{O}(\sigma, \rho ; \Gamma)$ will be the set of orbits induced by $\langle\sigma, \rho\rangle$. When the set $\Gamma$ is dropped from the expression, the set $\mathcal{O}(\sigma,[n])$ will be denoted $\mathcal{O}(\sigma)$.

$|\sigma|$ will denote the minimum number $m$ of transpositions $\tau_{1}, \ldots, \tau_{m}$ such that $\sigma=\tau_{1} \ldots \tau_{m}$; hence

$$
|\sigma|+|\mathcal{O}(\sigma)|=n
$$

The set $X_{\sigma}^{n}$ will denote the fixed point set under the action of $\sigma$ on $X^{n}$. Superscripts on $X$ will count the number of copies of itself on the cartesian 
product, and subscripts will be elements of the group and will determine fixed point sets.

For $A$ a graded ring and $m$ an integer, we will denote by $A[m]$ the ring $A$ whose grading is being shifted $m$ units to the left; in other words

$$
A[m]^{i}:=A^{i-m}
$$

The orbifold ring structure is easy to understand from $A\left(X^{n}, S_{n}\right)^{S_{n}}$ following the construction given previously. The only spaces left to study are the obstruction bundles and some splittings that occur using the orbits of the actions in $[n]$ of the elements of $S_{n}$.

Lemma 3.0.9. The orbifold shifting number of $\sigma \in S_{n}$ is

$$
\iota_{(\sigma)}=\sum_{j} n_{j} \frac{(j-1)}{2} N=\frac{N}{2}|\sigma|
$$

so that $2 \iota_{(\sigma)}=N|\sigma|$.

Proof. Once the action of $\sigma$ in $X^{n}$ is diagonalized every $j$-cycle has as eigenvalues all the $j$-roots of unity; the sum of these roots is $\frac{(j-1)}{2} N$.

As $|\sigma|+|\mathcal{O}(\sigma)|=n$ the shifting is taking into account that $X_{\sigma}^{n}$ is isomorphic to $X^{|\mathcal{O}(\sigma)|}$. Via the Kunneth isomorphism we have the following set of identities (everywhere the coefficient system for cohomology will be $\mathbb{C}$, so it will be dropped out of the notation)

$$
H^{*}\left(X_{\sigma}^{n}\right) \cong H^{*}(X)^{\otimes|\mathcal{O}(\sigma)|}
$$

then

$$
H^{*}(X)[N]^{\otimes|\mathcal{O}(\sigma)|} \cong H^{*}\left(X_{\sigma}^{n}\right)[|\mathcal{O}(\sigma)| \cdot N] \cong H^{*-2 \iota_{(\sigma)}}\left(X_{\sigma}^{n}\right)[n N]
$$

Using the notation of Lehn and Sorger [5, Section 2] where

$$
H^{*}(X)[N]\left\{S_{n}\right\}:=\bigoplus_{\sigma \in S_{n}} H^{*}(X)[N]^{\otimes|\mathcal{O}(\sigma)|} \cdot \sigma
$$

and the previous isomorphisms we get

Proposition 3.0.10. $H^{*}(X)[N]\left\{S_{n}\right\}$ and $A\left(X^{n}, S_{n}\right)[n N]$ are isomorphic as graded vector spaces. 
The ring structure of $H^{*}(X)[N]\left\{S_{n}\right\}$ is defined by the product (see [5])

$$
\begin{gathered}
m_{\pi, \rho}: H^{*}(X)[N]^{\otimes|\mathcal{O}(\pi)|} \otimes H^{*}(X)[N]^{\otimes|\mathcal{O}(\rho)|} \rightarrow H^{*}(X)[N]^{\otimes|\mathcal{O}(\pi \rho)|} \\
m_{\pi, \rho}(\alpha \otimes \beta)=f_{\langle\pi, \rho\rangle, \pi \rho}\left(f^{\pi,\langle\pi, \rho\rangle}(\alpha) \cdot f^{\rho,\langle\pi, \rho\rangle}(\beta) \cdot e^{g(\pi, \rho)}\right)
\end{gathered}
$$

where $g(\pi, \rho): \mathcal{O}(\pi, \rho) \rightarrow \mathbb{N}$ is the graph defect function; for $\Gamma \in \mathcal{O}(\pi, \rho)$

$$
g(\pi, \rho)(\Gamma)=\frac{1}{2}(|\Gamma|+2-|\mathcal{O}(\pi, \Gamma)|-|\mathcal{O}(\rho, \Gamma)|-\mid \mathcal{O}(\pi \rho, \Gamma))
$$

and

$$
e^{g(\pi, \rho)}:=\prod_{\Gamma \in \mathcal{O}(\pi, \rho)} e(X)^{g(\pi, \rho)(\Gamma)}
$$

where $e(X)$ is the Euler class of $X$.

For simplicity an abuse of notation is being made; the functions $f^{\pi,\langle\pi, \rho\rangle}$ are used to define either one of the following morphisms

$$
\begin{array}{ccc}
H^{*}\left(X_{\pi}^{n}\right) & \stackrel{f^{\pi,\langle\pi, \rho\rangle}}{\longrightarrow} & H^{*}\left(X_{\pi, \rho}^{n}\right) \\
\cong \uparrow & & \cong \uparrow \\
H^{*}(X)^{\otimes|\mathcal{O}(\pi)|} & \stackrel{f^{\pi,\langle\pi, \rho\rangle}}{\longrightarrow} & H^{*}(X)^{\otimes|\mathcal{O}(\pi, \rho)|} .
\end{array}
$$

It is clear that the product in $A\left(X^{n}, S_{n}\right)$ and in $H^{*}(X)[N]\left\{S_{n}\right\}$ are defined almost identically. The difference is on the last term, the Euler class of the obstruction bundle on one side and $e^{g(\pi, \rho)}$ on the other. We will see that these two terms represent the same class.

\subsection{The obstruction bundle.}

For $h_{1}, h_{2} \in S_{n}$ the obstruction bundle $E_{(\mathbf{h})}$ over $Y_{\left(h_{1}, h_{2}\right)}$ is defined by

$$
E_{(\mathbf{h})}=\left(H^{1}(\Sigma) \otimes e^{*} T Y\right)^{G}
$$

where $G=\left\langle h_{1}, h_{2}\right\rangle, Y=X^{n} / S_{n}$ and $\Sigma$ is a Riemann surface provided with a $G$ action such that $\Sigma / G=S_{\left(x_{1}, x_{2}, x_{3}\right)}^{2}$ is an orbifold Riemann sphere with three cone points points of angles $2 \pi / k_{i}$ where $k_{1}=\left|h_{1}\right|, k_{2}=\left|h_{2}\right|$ and $k_{3}=\left|h_{1} h_{2}\right|$ (as in section 2.3).

Because $H^{1}(\Sigma)$ is seen as a trivial bundle, the pullback of $E_{(\mathbf{h})}$ under $\pi: X_{h_{1}, h_{2}}^{n} \rightarrow Y_{\left(h_{1}, h_{2}\right)}$ is

$$
E_{h_{1}, h_{2}}:=\pi^{*} E_{(\mathbf{h})}=\left(H^{1}(\Sigma) \otimes \Delta^{*} T X^{n}\right)^{G}
$$


where $\Delta: X_{h_{1}, h_{2}}^{n} \hookrightarrow X^{n}$ is the inclusion (if $\rho: X^{n} \rightarrow Y$ is the quotient map, then $\rho \circ \Delta=e \circ \pi)$.

Without loss of generality we can assume that $\left|\mathcal{O}\left(h_{1}, h_{2}\right)\right|=k, n_{1}+\cdots+$ $n_{k}=n$ a partition of $n$ such that

$$
\Gamma_{i}=\left\{n_{1}+\cdots+n_{i-1}+1, \ldots, n_{1}+\cdots+n_{i}\right\}
$$

and $\left\{\Gamma_{1}, \Gamma_{2}, \ldots, \Gamma_{k}\right\}=\mathcal{O}\left(h_{1}, h_{2}\right)$. We will concentrate on each of the $\Gamma_{i}$ because we will see that the obstruction bundle $E_{h_{1}, h_{2}}$ can be seen as the product of $k$ bundles over $X$ (i.e. $E_{h_{1}, h_{2}}=\prod_{i} E_{h_{1}, h_{2}}^{i}$ ).

The following commutative diagrams
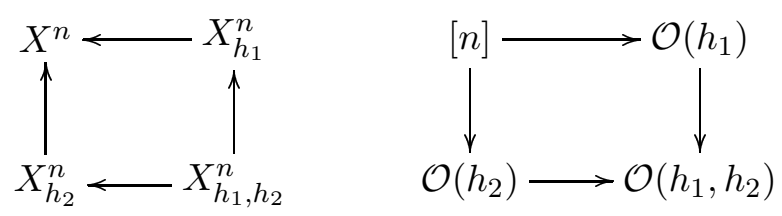

where arrows in the first one are inclusions of sets and the second are inclusion of orbits, induce the commutative diagram of diagonal inclusions

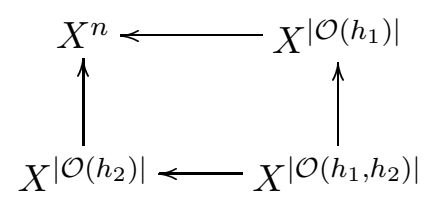

where every arrow is the product of the arrows of the following diagram for $i=1,2, \ldots, k$

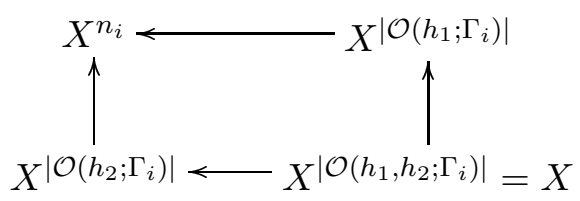

Lemma 3.1.1. For $\Delta_{i}: X \rightarrow X^{n_{i}} i=1, \ldots, k$ the diagonal inclusions, the bundles $\Delta_{i}^{*} T X^{n_{i}}$ become $G$ bundles via the restriction of the action of $G$ into the orbit $\Gamma_{i}$ and

$$
\Delta^{*} T X^{n} \cong \Delta_{1}^{*} T X^{n_{1}} \times \cdots \times \Delta_{k}^{*} T X^{n_{k}}
$$

as $G$ vector bundles.

Proof. This comes from the fact that the orbits $\Gamma_{i}$ are $G$ stable, hence $G$ induces an action on each $X^{n_{i}}$. 
Corollary 3.1.2. The obstruction bundle splits as

$$
E_{h_{1}, h_{2}}=\prod_{i=1}^{k}\left(H^{1}(\Sigma) \otimes \Delta_{i}^{*} T X^{n_{i}}\right)^{G}
$$

We can simplify the previous expression a bit further. Let $G_{i}$ be the subgroup of $S_{n_{i}}$ obtained from $G$ when its action is restricted to the elements in $\Gamma_{i}$; then we have a surjective homomorphism

$$
\lambda_{i}: G \rightarrow G_{i}
$$

where the action of $G$ into $\Delta_{i}^{*} T X^{n_{i}}$ factors through $G_{i}$. So we have

Lemma 3.1.3. $\left(H^{1}(\Sigma) \otimes \Delta_{i}^{*} T X^{n_{i}}\right)^{G} \cong\left(H^{1}(\Sigma)^{\operatorname{ker}\left(\lambda_{i}\right)} \otimes \Delta_{i}^{*} T X^{n_{i}}\right)^{G_{i}}$

Taking $\Sigma_{i}:=\Sigma / \operatorname{Ker}\left(\lambda_{i}\right)$ with the induced $G_{i}$ action we get that

$$
E_{h_{1}, h_{2}}^{i}:=\left(H^{1}\left(\Sigma_{i}\right) \otimes \Delta_{i}^{*} T X^{n_{i}}\right)^{G_{i}}
$$

hence

Proposition 3.1.4. The obstruction bundle splits as

$$
E_{h_{1}, h_{2}}=\prod_{i=1}^{k} E_{h_{1}, h_{2}}^{i}
$$

As the action of $G_{i}$ in $\Delta_{i}^{*} T X^{n_{i}}$ is independent on the structure of $X$ (moreover, it depends only in the coordinates), hence

$$
\Delta_{i}^{*} T X^{n_{i}} \cong T X \otimes \mathbb{C}^{n_{i}}
$$

as $G_{i}$-vector bundles, where $T X$ is the tangent bundle over $X$ and $G_{i} \subset S_{n_{i}}$ acts on $\mathbb{C}^{n_{i}}$ in the natural way. Then

\section{Lemma 3.1.5.}

$$
E_{h_{1}, h_{2}}^{i} \cong T X \otimes\left(H^{1}\left(\Sigma_{i}\right) \otimes \mathbb{C}^{n_{i}}\right)^{G_{i}}
$$

Defining $r\left(h_{1}, h_{2}\right)(i):=\operatorname{dim}_{\mathbb{C}}\left(H^{1}(\Sigma) \otimes \mathbb{C}^{n_{i}}\right)^{G_{i}}$ it follows that the Euler class of $E_{h_{1}, h_{2}}^{i}$ equals the Euler class of $X$ to some exponent

Corollary 3.1.6. $c\left(E_{h_{1}, h_{2}}^{i}\right)=e(X)^{r\left(h_{1}, h_{2}\right)(i)}$ 
in other words,

$$
c\left(E_{h_{1}, h_{2}}^{i}\right)=\left\{\begin{array}{cc}
1 & \text { if } r\left(h_{1}, h_{2}\right)(i)=0 \\
e(X) & \text { if } r\left(h_{1}, h_{2}\right)(i)=1 \\
0 & \text { if } r\left(h_{1}, h_{2}\right)(i) \geq 2
\end{array}\right.
$$

Making it clear that the obstruction looks like

$$
c\left(E_{h_{1}, h_{2}}\right)=\prod_{i=1}^{k} e(X)^{r\left(h_{1}, h_{2}\right)(i)}
$$

Proposition 3.1.7. $H^{*}(X)[N]\left\{S_{n}\right\}$ and $A\left(X^{n}, S_{n}\right)[n N]$ are isomorphic as rings.

Proof. The only thing left to prove is that the graph defect function $g\left(h_{1}, h_{2}\right)$ matches the function $r\left(h_{1}, h_{2}\right)$ just defined. Working on the orbifold cup product, using the previous commutative diagrams (especially 3.2) and the splittings we can restrict ourselves to each orbit $\Gamma_{i}$ of $\mathcal{O}\left(h_{1}, h_{2}\right)$, hence for

$$
\begin{gathered}
\alpha \in H^{p-2 \iota\left(h_{1} \Gamma_{\Gamma_{i}}\right)}\left(X^{\left|\mathcal{O}\left(h_{1} ; \Gamma_{i}\right)\right|}\right) \\
\beta \in H^{q-2 \iota\left(h_{2} \Gamma_{\Gamma_{i}}\right)}\left(X^{\left|\mathcal{O}\left(h_{2} ; \Gamma_{i}\right)\right|}\right) \\
\alpha \in H^{r-2 \iota\left(\left(h_{1} h_{2}\right)^{-1} \Gamma_{\Gamma_{i}}\right)}\left(X^{\left|\mathcal{O}\left(\left(h_{1} h_{2}\right)^{-1} ; \Gamma_{i}\right)\right|}\right)
\end{gathered}
$$

with $p+q+r=2 n_{i} N$, and $\left|\Gamma_{i}\right|=n_{i}$

$$
\begin{aligned}
\operatorname{deg} \alpha+\operatorname{deg} \beta+\operatorname{deg} \gamma & =p+q+r-2\left(\iota_{\left(h_{1} \Gamma_{\Gamma_{i}}\right)}+\iota_{\left(h_{2} \Upsilon_{\Gamma_{i}}\right)}+\iota_{\left(\left(h_{1} h_{2}\right)^{-1} \Gamma_{\Gamma_{i}}\right)}\right) \\
& =\left(2 n_{i}-\mid h_{1}\left\lceil\Gamma _ { i } | - | h _ { 2 } \left\lceil\Gamma_{i}|-| h_{1} h_{2}\left\lceil\Gamma_{i} \mid\right) N\right.\right.\right. \\
& =\left(\left|\mathcal{O}\left(h_{1} ; \Gamma_{i}\right)\right|+\left|\mathcal{O}\left(h_{2} ; \Gamma_{i}\right)\right|+\left|\mathcal{O}\left(h_{1} h_{2} ; \Gamma_{i}\right)\right|-n_{i}\right) N
\end{aligned}
$$

so we get that

$$
\begin{aligned}
\operatorname{dim}_{\mathbb{R}} E_{h_{1}, h_{2}}^{i} & =\operatorname{dim}_{\mathbb{R}} X-\operatorname{deg} \alpha-\operatorname{deg} \beta-\operatorname{deg} \gamma \\
& =\frac{1}{2}\left(2+n_{i}-\left|\mathcal{O}\left(h_{1} ; \Gamma_{i}\right)\right|-\left|\mathcal{O}\left(h_{2} ; \Gamma_{i}\right)\right|-\left|\mathcal{O}\left(h_{1} h_{2} ; \Gamma_{i}\right)\right|\right) 2 N
\end{aligned}
$$

which implies that

$$
r\left(h_{1}, h_{2}\right)(i)=\frac{1}{2}\left(2+n_{i}-\left|\mathcal{O}\left(h_{1} ; \Gamma_{i}\right)\right|-\left|\mathcal{O}\left(h_{2} ; \Gamma_{i}\right)\right|-\left|\mathcal{O}\left(h_{1} h_{2} ; \Gamma_{i}\right)\right|\right)
$$


matching precisely the definition of the graph defect function $g\left(h_{1}, h_{2}\right)$ (see formula 3.1).

Using the notation of $[5]$

$$
H^{*}(X)[N]^{[n]}:=\left(H^{*}(X)[N]\left\{S_{n}\right\}\right)^{S_{n}}
$$

we can conclude

Theorem 3.1.8. For $X$ a compact complex even dimensional manifold $\left(\operatorname{dim}_{\mathbb{C}}(X)=N\right)$ we have that

$$
H_{\text {orb }}^{*}\left(X^{n} / S_{n} ; \mathbb{C}\right)[n N] \cong H^{*}(X)[N]^{[n]}
$$

Proof. The rings $H^{*}(X)[N]\left\{S_{n}\right\}$ and $A\left(X^{n}, S_{n}\right)[n N]$ are isomorphic and they have a compatible $S_{n}$ equivariant action, hence their invariant rings are also isomorphic, so

$$
H_{\text {orb }}^{*}\left(X^{n} / S_{n} ; \mathbb{C}\right)[n N] \cong A\left(X^{n}, S_{n}\right)[n N]^{S_{n}} \cong\left(H^{*}(X)[N]\left\{S_{n}\right\}\right)^{S_{n}}
$$

\subsection{Hilbert Schemes.}

Now we are ready to prove a conjecture posed by Ruan [7, Conj. 6] about algebraic surfaces. Let $X$ be a smooth projective surface over the complex numbers and $X^{[n]}$ its $n$-th Hilbert scheme of points of length $n ; X^{[n]}$ is again projective and smooth of dimension $2 n$. Lehn and Sorger proved

Theorem 3.2.1. [5, Thm. 3.2] Let $X$ be a smooth projective surface with trivial canonical divisor. Then there is a canonical isomorphism of graded rings

$$
\left(H^{*}(X ; \mathbb{C})[2]\right)^{[n]} \cong H^{*}\left(X^{[n]} ; \mathbb{C}\right)[2 n]
$$

Remark 3.2.2. This isomorphism is obtained after changing the sign on the integral over the fundamental homology class of $X$. In the notation of [5] $T(a):=-\int_{[X]} a$, for $a \in H^{*}(X ; \mathbb{C})$.

Using the results of the previous section we obtain a positive answer to the conjecture 
Theorem 3.2.3. Let $X$ be a smooth projective surface with trivial canonical divisor. Then there is a canonical isomorphism (up to a change of sign) of graded rings between the orbifold cohomology of $X^{n} / S_{n}$ and the cohomology of the $n$-th Hilbert scheme of $X$

$$
H_{\text {orb }}^{*}\left(X^{n} / S_{n} ; \mathbb{C}\right) \cong H^{*}\left(X^{[n]} ; \mathbb{C}\right)
$$

Proof. By theorems 3.2.1 and 3.1.8

$$
H_{\text {orb }}^{*}\left(X^{n} / S_{n} ; \mathbb{C}\right)[2 n] \cong\left(H^{*}(X ; \mathbb{C})[2]\right)^{[n]} \cong H^{*}\left(X^{[n]} ; \mathbb{C}\right)[2 n]
$$

\section{References.}

[1] Michael Atiyah and Graeme Segal, On equivariant Euler characteristics, J. Geom. Phys. 6 (1989), no. 4, 671-677.

[2] Weimin Chen and Yongbin Ruan, A New Cohomology Theory for Orbifold, arXiv:math.AG/0004129.

[3] L. Dixon, J. A. Harvey, C. Vafa, and E. Witten, Strings on orbifolds, Nuclear Phys. B 261 (1985), no.4, 678-686.

[4] Barbara Fantechi and Lothar Göttsche, Orbifold Cohomology for Global Quotients, arXiv:math.AG/0104207.

[5] Manfred Lehn and Christoph Sorger, The Cup Product of the Hilbert Scheme for K3 surfaces, arXiv:math.AG/0012166.

[6] Manfred Lehn and Christoph Sorger, Symmetric groups and the cup product on the cohomology of Hilbert schemes, Duke Math. J. 110 (2001), no. $2,345-357$.

[7] Yongbin Ruan, Stringy geometry and topology of orbifolds, Symposium in Honor of C. H. Clemens (Salt Lake City, UT, 2000), Contemp. Math., vol. 312, Amer. Math. Soc., Providence, RI, 2002, pp. 187-233.

[8] Peter Scott, The geometries of 3-manifolds, Bull. London Math. Soc. 15 (1983), no. 5, 401-487.

[9] Eric Zaslow, Topological orbifold models and quantum cohomology rings, Comm. Math. Phys. 156 (1993), no. 2, 301-331. 


\begin{abstract}
Mathematics Department UNIVERSITY OF WISCONSIN MAdison, Wisconsin, 53706 uribe@math . wisc.edu
\end{abstract}

Received March 27, 2002. 